\title{
Fuzzy logic based monitoring system for detecting radon concentration
} Nilesh Dashore and Gopal Upadhyay ${ }^{2}$

\author{
${ }^{1}$ Dept. of Physics, B.M Engg. College, Indore; ' ${ }^{2}$ School of Studies in Physics, Vikram University, Ujjain, India \\ nk_dashore@yahoo.co.in, gopalujjain@yahoo.co.in
}

Abstract: This paper describes a fuzzy logic based Radon monitoring system able to detect, count and store the amount of alpha particles produced by radioactive decay of environmental Radon-222.We applied our suggested model to sensors network. The mobile sensor network is composed of a collection of distributed nodes each of which has limited sensing, intelligence and communication capabilities. An adhoc wireless network is established among all nodes and each node considers other node as extended sensor.

Keywords: Radon monitoring system, Fuzzy logic control. Introduction

Radon is a natural radio active gas, which occurs naturally in all rocks and solids. Radon and its decay products called Radon progeny emit highly ionizing alpha radiation (WHO, 2004). Decay products are suspended in the air which we breathe. In places such as mines, caves it can accumulate up to dangerous concentrations and may cause substantial health damage after long term exposure. Nowadays it is well known that the risk of developing lung cancer rises with the level of radon and the exposition time. Radon concentration in the air is measured as the amount of radioactivity $(\mathrm{Bq})$ in a cubic meter of air $\left(\mathrm{Bq} / \mathrm{m}^{3}\right)$. One Becquerel $(\mathrm{Bq})=1$ disintegration of atom per second.

\section{Radon monitoring system}

Radon monitoring system is a real time remote sensing system which is able to detect, count and store the amount of alpha particles produced by the decay of environmental Radon-222. R.Pena-Eguiluz ${ }^{1}$ suggested a continuous Radon monitoring system based on a solid state silicon detector (Pena Eguiluz et al., 2007). This system is built from a Discriminating Industrial alpha detector.

The system has been conceived as a remote sensing unit, so it must be able to work alone and in adverse environments. As a consequence, most of the system components are protected against outdoor conditions by a stainless steel sealed tube case. At one of its ends a protected detection radon chamber is located, where the environmental air can reach the semiconductor detector. At the top of the tube there is a sealed connector which allows to supply the voltage from the battery and to establish a serial communication with an external computer.

\section{Wireless sensor and adhoc network}

Wireless sensor networks generally consist of a data acquisition network and a data distribution network, monitored and controlled by a management center.

Wireless sensor network (WSN) is the most potential technology for very low power ubiquitous networks. Foreseen applications fields include monitoring of remote or hostile geographical regions. WSN may consist of

Research article

CIndian Society for Education and Environment (iSee) even thousands of small and fully autonomous nodes, which gather sensor information, perform data processing, and communicate with each other (Cui et al., 2004). Sensor networks are the key to gathering the information needed by smart environments, whether in mines, caves buildings, utilities, industrial, home, or elsewhere. A general setup of a wireless sensor network consists of a large number of sensors randomly and densely deployed in a certain area. Each compact sensor usually is capable of sensing, processing data at a small scale, and communicating through Omni-directional radio signal. An ad hoc network is formed by wireless mobile nodes (hosts) that operate as terminals as well as routers in the network, without any centralized administration (Lewis, 2005). Mobile ad hoc networks provide temporary wireless networking capability in situations where no fixed infrastructure exists. These networks are created spontaneously without any infrastructure. Since an ad hoc network can be deployed rapidly with relatively low cost, it becomes an attractive option for commercial uses such as sensor networks or virtual classrooms.

Wireless ad-hoc sensor networks have recently attracted much interest in the wireless research community as a fundamentally new tool for a wide range of monitoring and data-gathering applications (Mohammad Abdul Azim \& Abbas Jamlipour, 2006).

\section{Block diagram of fuzzy sensor}

Fig. 1 shows the block diagram of fuzzy sensor in which we show that sensor inputs are given to fuzzy controller. Where the sensor inputs supplied for fuzzification and then Inference engine is used to find out rule based output and after defuzzification crisp output is controlled to get sensor deployment location (Dashore Pankaj \& Jain Suresh, 2008).

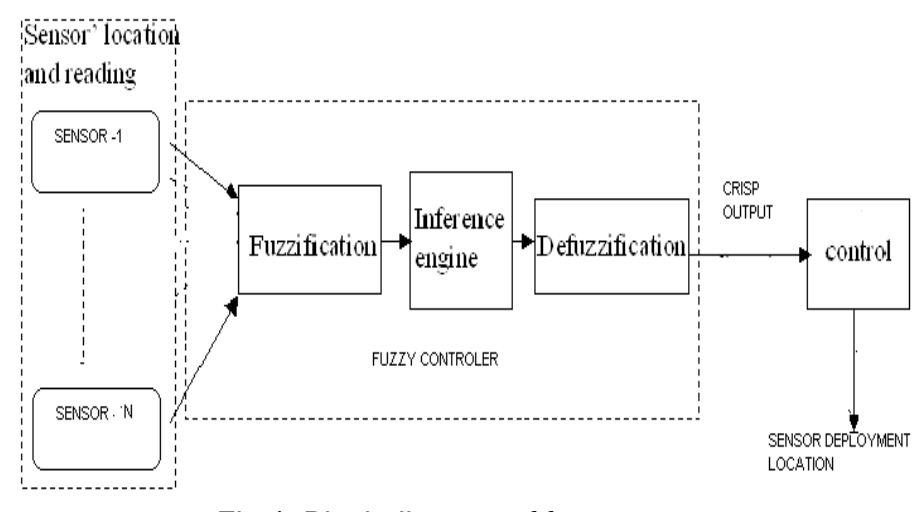

Fig. 1. Block diagram of fuzzy sensor

Fuzzy logic control process to detect the radon concentration

Fuzzyfication: Comprises the process of transforming crisp values into grades of membership for linguistic 
terms of fuzzy sets. The membership function is used to associate a grade to each linguistic term. The fuzzification is the first step in fuzzy logic processing involves a domain transformation where the crisp inputs are transformed into fuzzy inputs. To transform crisp inputs into fuzzy inputs, membership function must first be determined for each point.

Fig. 2 shows the membership function graph for location angle of sensors, where 8 types of linguistic variables are used as the inputs of fuzzy wireless sensor with related location angle of sensors as $X A, X B, X C, X D, X E, X F, X G, X H$. The set of sensor values switched at different angles are provides the Input values. The set of sensor input values are given for fuzzification through membership functions.

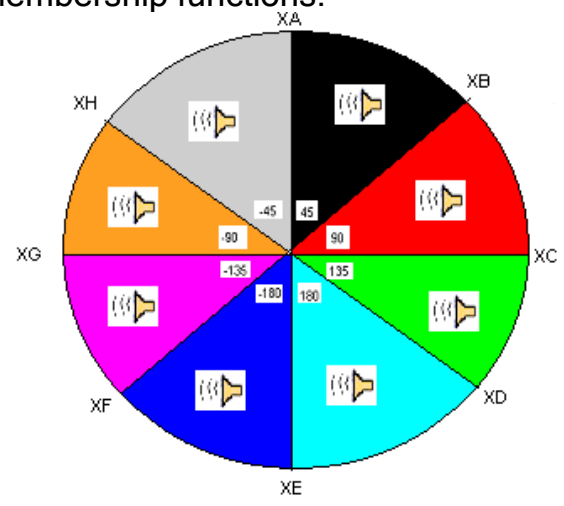

Fig.2. Location angle of sensors

The membership function for the location angle set and sensor moving direction set are shown in Fig. 3. The sensor location angles range from $-180^{\circ}$ to $+180^{\circ}$. The membership function of concentration set is shown in Fig. 4 at low, medium and high values between 0 and 1 .

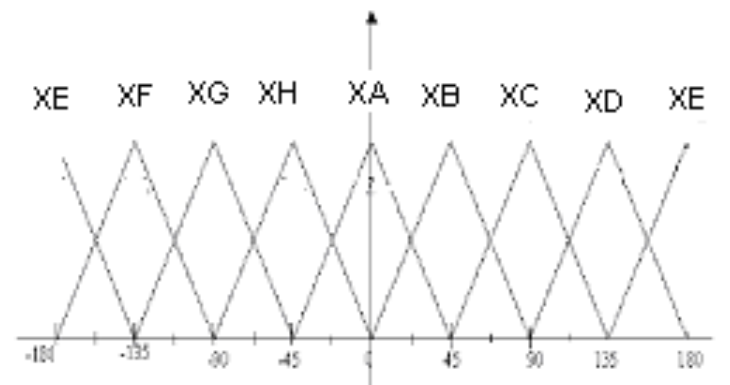

Fig.3. Membership function for location angle of sensors

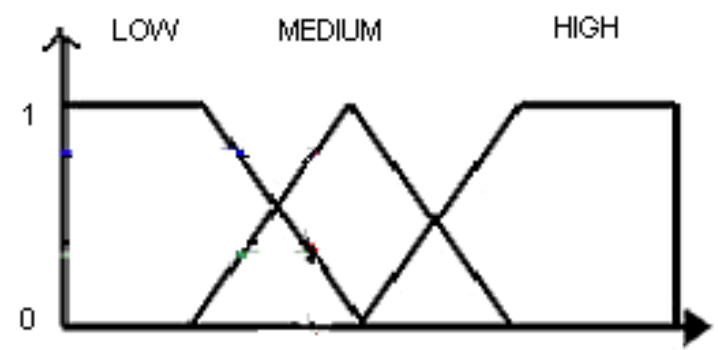

Fig.4. Membership function for concentration

Research article

CIndian Society for Education and Environment (iSee)
Vol.2 No 5 (May 2009)

ISSN: 0974- 6846

Fuzzy controller. Evaluates the fuzzy rules with the fuzzy inputs. Fuzzy controller needs to convert the input information into nodes next movement direction (Dashore Pankaj \& Jain Suresh, 2008). The natural diffusion phenomena of gases and aerosols tend to spread in the environment inducing a concentration gradient that can be used as a clue for tracing emission sources. The rule set should tend to steer all nodes to the vicinity of the highest reported concentration. In this study MATLAB fuzzy logic tool box was used by us to generate FLC module. These fuzzy rules are described in IF-THEN form and use the linguistic variables:

IF concentration is $\mathrm{HIGH}$ and direction is XA THEN move to $X A$ expansion cell.

IF concentration is $\mathrm{HIGH}$ and direction is $\mathrm{XB}$ THEN move to $X B$ expansion cell.

IF concentration is $\mathrm{HIGH}$ and direction is $\mathrm{XH}$ THEN move to $\mathrm{XH}$ expansion cell.

Defuzzification: Since the task of the FLC controller is steering the node's sensor towards the highest reported concentration in our MATLAB flc module, the centre of gravity method is used to get a crisp output to control the next moving direction. The FLC controller uses other nodes current location to find out all expansion cells the node has and uses the moving direction generated by the FLC to define the expansion cell located on the path of the moving direction as the nodes optimal deployment location.

Conclusion

Fuzzy logic system was used to detect the concentration of Radon. A wireless sensor network was employed to search for higher concentration of Radon. This approach ensures that all nodes of the network maintain a dynamically stable adhoc wireless communication network for collaborative exploration and information fusion on Radon concentration.

\section{References}

1. Pena Eguiluz R, Garcia Hernandez JM and Ramirez Jimenez FJ (2007) A continuous radon monitoring system based on a solid-state silicon detector. J. Appl. Res. Technol.April volume 5 pp 3-9

2. WHO (2004) www.who.int/entity/phe/radiation/ en/2004Radon.pdf.

3. Cui X, Hardin T, Ragade RK and Elmaghraby AS (2004 IEEE International Conference on Volume, Issue , 25-27 Oct. 2004 Page(s): 194 - 203) A Swarm-based Fuzzy Logic Control Mobile Sensor Network for Hazardous Contaminants Localization. University of Louisville, KY40292.

4. Mohammad Abdul Azim and Abbas Jamlipour (2006) Optimized forwarding for wireless sensor networks by fuzzy inference system. The University of Sydney, NSW 2006, Australia.

5. Lewis FL (2005) Wireless Sensor Network. The University of Texas, Arlington.

6. Dashore Pankaj and Jain Suresh (2008) Hybrid Rulebased Fuzzy logic system for decision making. IEEE World Congress WCCi-2008.

"Radon detection" http://www.indjst.org

Dashore \& Upadhyay Indian J.Sci.Technol. 